\title{
Can Water Temperature and Immersion Time Influence the Effect of Cold Water Immersion on Muscle Soreness? A Systematic Review and Meta-Analysis
}

\author{
Aryane Flauzino Machado ${ }^{1}$ Paulo Henrique Ferreira ${ }^{2} \cdot$ Jéssica Kirsch Micheletti $^{1}$. \\ Aline Castilho de Almeida ${ }^{3}$. Ítalo Ribeiro Lemes ${ }^{1} \cdot$ Franciele Marques Vanderlei $^{1}$. \\ Jayme Netto Junior ${ }^{1}$ Carlos Marcelo Pastre ${ }^{1}$
}

Published online: 18 November 2015

(C) Springer International Publishing Switzerland 2015

\begin{abstract}
Background Cold water immersion (CWI) is a technique commonly used in post-exercise recovery. However, the procedures involved in the technique may vary, particularly in terms of water temperature and immersion time, and the most effective approach remains unclear.

Objectives The objective of this systematic review was to determine the efficacy of CWI in muscle soreness management compared with passive recovery. We also aimed to identify which water temperature and immersion time provides the best results.

Methods The MEDLINE, EMBASE, SPORTDiscus, PEDro [Physiotherapy Evidence Database], and CENTRAL (Cochrane Central Register of Controlled Trials) databases were searched up to January 2015. Only randomized controlled trials that compared CWI to passive recovery were included in this review. Data were pooled in a meta-analysis and described as weighted mean differences (MDs) with $95 \%$ confidence intervals (CIs).
\end{abstract}

Electronic supplementary material The online version of this article (doi:10.1007/s40279-015-0431-7) contains supplementary material, which is available to authorized users.

Carlos Marcelo Pastre

pastre@fct.unesp.br

1 Departamento de Fisioterapia, Faculdade de Ciências e Tecnologia, Universidade Estadual Paulista, 305 Roberto Simonsen, Presidente Prudente, São Paulo 19060-900, Brazil

2 Discipline of Physiotherapy, Faculty of Health Science, The University of Sydney, Sydney, NSW, Australia

3 Departamento de Fisioterapia, Centro de Ciências Biológicas e da Saúde, Universidade Federal de São Carlos, São Carlos, Brazil
Results Nine studies were included for review and metaanalysis. The results of the meta-analysis revealed that CWI has a more positive effect than passive recovery in terms of immediate (MD $=0.290,95 \%$ CI 0.037, 0.543; $p=0.025)$ and delayed effects $(\mathrm{MD}=0.315,95 \% \mathrm{CI}$ $0.048,0.581 ; p=0.021)$. Water temperature of between 10 and $15{ }^{\circ} \mathrm{C}$ demonstrated the best results for immediate $(\mathrm{MD}=0.273,95 \%$ CI $0.107,0.440 ; p=0.001)$ and delayed effects $(\mathrm{MD}=0.317,95 \%$ CI $0.102,0.532$; $p=0.004)$. In terms of immersion time, immersion of between 10 and $15 \mathrm{~min}$ had the best results for immediate $(\mathrm{MD}=0.227,95 \% 0.139,0.314 ; p<0.001)$ and delayed effects $(\mathrm{MD}=0.317,95 \% 0.102,0.532, p=0.004)$.

Conclusions The available evidence suggests that CWI can be slightly better than passive recovery in the management of muscle soreness. The results also demonstrated the presence of a dose-response relationship, indicating that $\mathrm{CWI}$ with a water temperature of between 11 and $15{ }^{\circ} \mathrm{C}$ and an immersion time of 11-15 min can provide the best results.

\section{Key Points}

Cold water immersion (CWI) can be slightly better than passive recovery in management of muscle soreness.

The findings suggest a dose-response relationship, indicating that CWI at a temperature between 11 and $15{ }^{\circ} \mathrm{C}$ for $11-15$ min can provides the best results for both immediate and delayed effects.

A potential risk of bias was identified by methodological quality assessment of the studies included, identifying a need for higher-quality studies to affirm that the dose-response relationship of the results can be reliably reproduced. 


\section{Background}

Several post-exercise recovery techniques are currently employed in an attempt to return the body to its preexercise state $[1,2]$. Cold water immersion (CWI) has become popular in sports [3, 4] as it is a low-cost technique that is easily performed in different situations [5] and has been found to alleviate physiological and functional deficits associated with exercise-induced muscle damage [6]. Compared with controlled interventions and other traditional recovery techniques, CWI achieves positive muscle soreness reduction results following a range of exercise types [7, 8]. Yet the specific mechanisms associated with CWI response are unknown $[9,10]$.

Despite its widespread use, significant procedural variations in CWI exist $[11,12]$. Investigations have suggested that physiological changes are temperature dependent [13], causing alterations in the body [14]. However, other factors may influence recovery. Studies claim that the magnitude of these mechanisms depends on the intensity of the cold and how it affects the body [15]. Pastre et al. [16] attribute response variation to differences in the application of CWI, such as water temperature, immersion time and type of CWI, being able to cause changes in blood flow [14], metabolic activity [17], and nerve conduction velocity (NCV) [18].

In recent years, the number of studies focusing on CWI has increased, and major systematic reviews have been performed to compare the effects of CWI and other soreness recovery strategies $[8,12]$. However, the focus of these systematic reviews $[8,12]$ was to analyze the effects of CWI on muscle soreness, regardless of their application strategy. Thus, the dose-response relationship, aimed at finding the best dosage, including analysis of temperature and duration of immersion, has not yet been investigated. Glasgow et al. [10] showed that studies focusing on different CWI application strategies can contribute to determining the risks and benefits for athletes.

A systematic review involving the dose-response relationship will clarify the most effective method of application of CWI for post-exercise muscle soreness. Therefore, the purpose of this systematic review was to determine the efficacy of CWI on the management of muscle soreness compared with control intervention (passive recovery). An analysis of which dosage of application provides the best results, focusing on water temperature and immersion time, was also undertaken.

\section{Methods}

This systematic review was registered in an international database of systematic reviews in health and social care (registration number CRD42015016573; http://www.crd. york.ac.uk/PROSPERO/).

\subsection{Search Strategies}

Studies were selected after searching five databases [MEDLINE, EMBASE, SPORTDiscus, PEDro (Physiotherapy Evidence Database), and CENTRAL (Cochrane Central Register of Controlled Trials)] from the earliest record of each database to 21 January 2015. The terms and keywords used to search optimization were related to randomized controlled trials: cold water immersion and post-exercise recovery (see details in Electronic Supplementary Material Appendix 1). The reference list of eligible clinical trials was searched by hand to complement the electronic searches. No restrictions were applied to the sample conditions (age, sex, exercise level) or language of the studies.

\subsection{Study Selection}

The studies selected involved CWI treatment with human participants and assessed post-exercise muscle soreness. CWI was defined as immersion in water with a temperature $\leq 15{ }^{\circ} \mathrm{C}[5,11,14]$. To be eligible, studies had to (1) be randomized controlled trials comparing CWI and control condition post-exercise; (2) be studies that used a single session of exercises; (3) apply CWI within $1 \mathrm{~h}$ of the end of the exercise; and (4) include only one immersion on the first day. Studies using intermittent immersions or more than one immersion on subsequent days were excluded. The control condition was considered as passive recovery, in which the subjects remained seated, without any attempt to accelerate recovery [1]. Exercise protocols performed on a single day were considered as a single exercise session, regardless of the duration or types of exercises.

The study selection process was conducted by title, followed by abstract, and full text (Fig. 1). These steps were performed independently by two authors (ACA and JKM) and consensus was used to solve disagreements.

\subsection{Data Extraction}

Outcome data, including final values of means and standard deviations and sample size were extracted by two review- 
Fig. 1 Flow chart for selection of studies. CENTRAL Cochrane Central Register of Controlled Trials, $C W I$ cold water immersion, PEDro

Physiotherapy Evidence Database

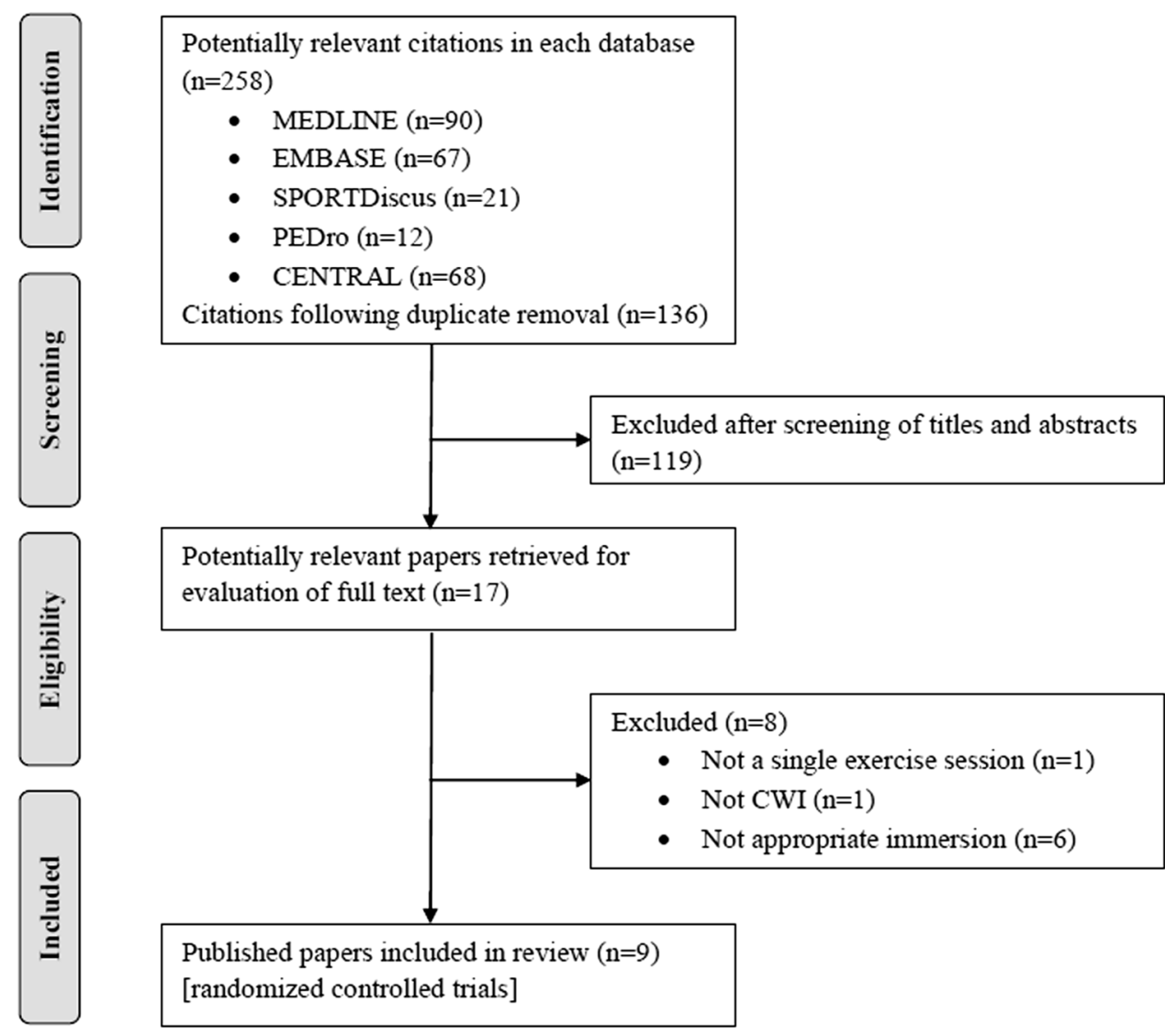

ers (AFM, JKM). The data extraction process was performed using a standardized form that included details such as characteristics of participants, exercise procedures, CWI procedures, outcome measures, and methodological characteristics. Disagreements between authors regarding data extraction were resolved by consensus.

Some studies included multiple observations. In such cases, data were extracted at a clinically relevant timepoint in order to analyze immediate effects (up to $24 \mathrm{~h}$ postexercise) and delayed effects (after $24 \mathrm{~h}$ post-exercise). For the delayed effects, the peak soreness of the control group was considered in order to minimize interference caused by the intervention. Soreness scores were converted to a common 0-10 scale.

\subsection{Quality Assessment}

All studies included were assessed for methodological quality. This process was performed by two independent reviewers (AFM and JKM) using the PEDro scale [19, 20]. Each study was assessed for random allocation, concealed allocation, baseline comparability, blinding participants, therapists and assessors, adequate follow-up, intention-totreat, between-group comparison, point estimates, and variability. A score $\geq 7$ was considered 'high quality', a score of 5 or 6 was considered 'moderate quality', and $\leq 4$ was considered 'poor quality'. If trials had already been assessed and listed on the PEDro database, such scores were adopted. Methodological quality was not an inclusion criterion.

\subsection{Data Synthesis}

Analyses of the temperature and immersion time were performed separately. The stratification process was based on two aspects: physiological and information on previous studies. Regarding the physiological aspect, the relationship between exposure to cold and changes in NCV [18] and blood flow [14] are described but the magnitude remains uncertain with small variations [21]. Regarding the characteristics of previous studies, Bleakley et al. [12] observed that approximately $75 \%$ of studies involving CWI used water temperatures between 10 and $15{ }^{\circ} \mathrm{C}$ and the average of time of exposure was approximately $12 \mathrm{~min}$. Thus, after verifying similar values to those found by the authors, and due to the interest in exploring outcomes from a range of temperatures and times, the following criteria were established:

- A median of $12{ }^{\circ} \mathrm{C}$ was observed for water temperature. Studies with water temperatures below the median, temperatures of 5 and $10{ }^{\circ} \mathrm{C}$, were categorized as 
'severe cold' $\left(5-10^{\circ} \mathrm{C}\right)$. Studies with temperatures above $10{ }^{\circ} \mathrm{C}$ were categorized as 'moderate cold' $\left(11-15^{\circ} \mathrm{C}\right)$.

- For immersion time, a median of 14 min was observed. Studies that used immersion times below the median, 5 and $10 \mathrm{~min}$, were categorized as 'short immersion' (5-10 min). The remainder of the studies used a CWI of $14 \mathrm{~min}$, except for two studies that used 15 and $20 \mathrm{~min}$. Thus, studies with immersion times of up to $15 \mathrm{~min}$ (11-15 min) were categorized as 'medium immersion' and studies with an immersion time above $15 \mathrm{~min}$ (16-20 min) as 'longer immersion', due to amplitude of times.

It is noteworthy that the entire descriptive analysis process was conducted prior to execution of the metaanalysis.

\subsection{Data Analysis}

Comprehensive Meta-Analysis software, version 2.2.04 (Biostat, Englewood, NJ, USA) was used for all analysis and pooled estimates were calculated using a random-effect model, due to the heterogeneity of the studies (represented by $I^{2}$ ). Data were pooled in meta-analyses and described as weighted mean differences (MDs) with $95 \%$ confidence intervals (CIs). The immediate and delayed effects were calculated in order to analyze the effect of CWI, independent of water temperature and duration of immersion. In case of more than one intervention group per study, the group that represented the lowest effect size was used.

\section{Results}

The database search identified 258 studies and 17 were chosen for full-text review. Of these articles, eight were excluded: one was not a single exercise session, one used a cryotherapy technique other than CWI, and six did not feature an appropriate immersion based on the inclusion criteria. Figure 1 shows the schematic process of the study selection based on a PRISMA flow diagram.

Assessment of the methodological quality of the studies included using the PEDro scale reported a mean of 4.2. Three studies [22-24] were considered as 'moderate quality' and another six studies as 'poor quality' [2, 9, 2528 ]. Due to the type of intervention, blinding was often not possible but $44.4 \%$ of the studies described adequate follow-up procedures (see details in Electronic Supplementary Material Appendix 2). Figure 2 shows the number of clinical trials that fulfilled each criterion.

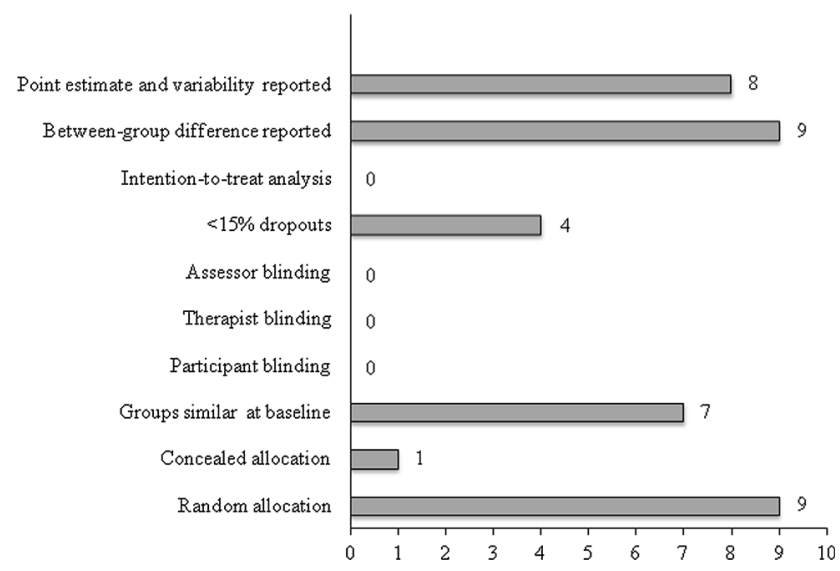

Fig. 2 Number of trials meeting individual PEDro [Physiotherapy Evidence Database] criteria

The nine eligible studies were published between 2007 [9] and 2015 [22]. These studies comprised a total of 169 participants (male, $n=141$; female, $n=28$ ). The health conditions of the participants, i.e., the level of exercise, fluctuated between physically active and athletes.

The studies were from Australia [2, 23, 24, 27], the UK [9, 22, 28], and the USA [25, 26]. All were randomized controlled trial-type studies, while six were parallel-group trials [9, 22, 25-28], and three used a crossover design [2, $23,24]$. Exercise protocols consisted primarily of exercises that required high physical ability with possible subsequent onset of soreness, such as shuttle running [9, 22], downhill running [25], an Australian Football match and training [23, 27], high-intensity intervals [2, 24], and countermovement jumps [28].

Interventions were varied. Water temperature ranged from 5 [25] to $15^{\circ} \mathrm{C}$ [2] and immersion time varied between 5 [24] and 20 [25] min. Eight studies [2, 9, 22-24, 26-28] used passive recovery in which participants had to remain seated with minimal movement, while one did not report such information [25]. Immersion depth ranged from immersion of the lower limbs [9, 25, 28] to immersion of the whole body, excluding only the head and neck [24]. It was observed that seven [2, 9, 22, 23, 25-27] of eight studies that evaluated delayed effect found peak soreness at $24 \mathrm{~h}$ post-exercise, and only one [23] found peak soreness at $48 \mathrm{~h}$ post-exercise.

The characteristics of the included studies are summarized in Table 1.

\subsection{Analysis of Water Temperature}

Seven studies [9, 23-28] provided data related to the immediate effects of CWI. The subgroup analysis of the pooled results is shown in Fig. 3. A general analysis of the 


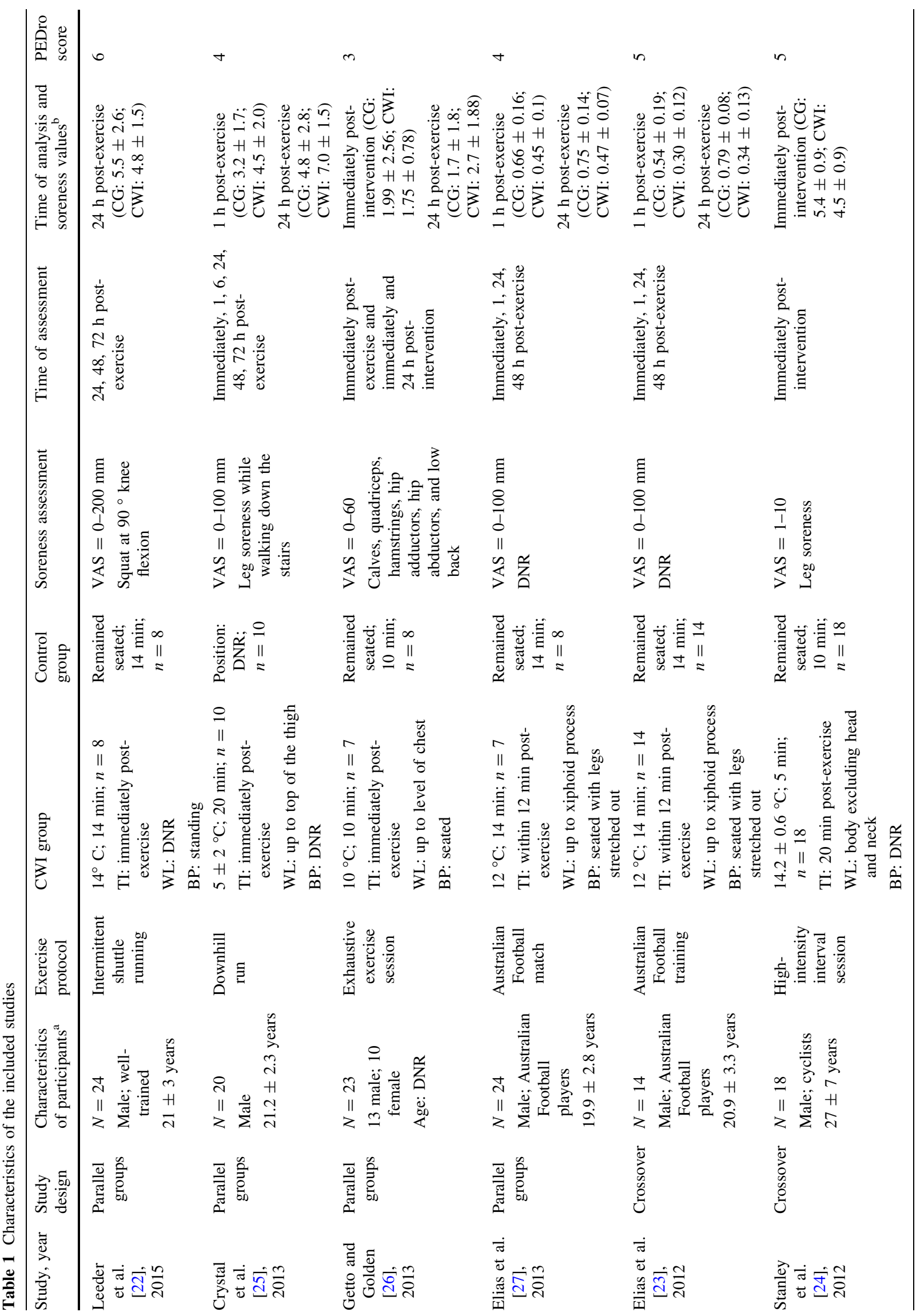




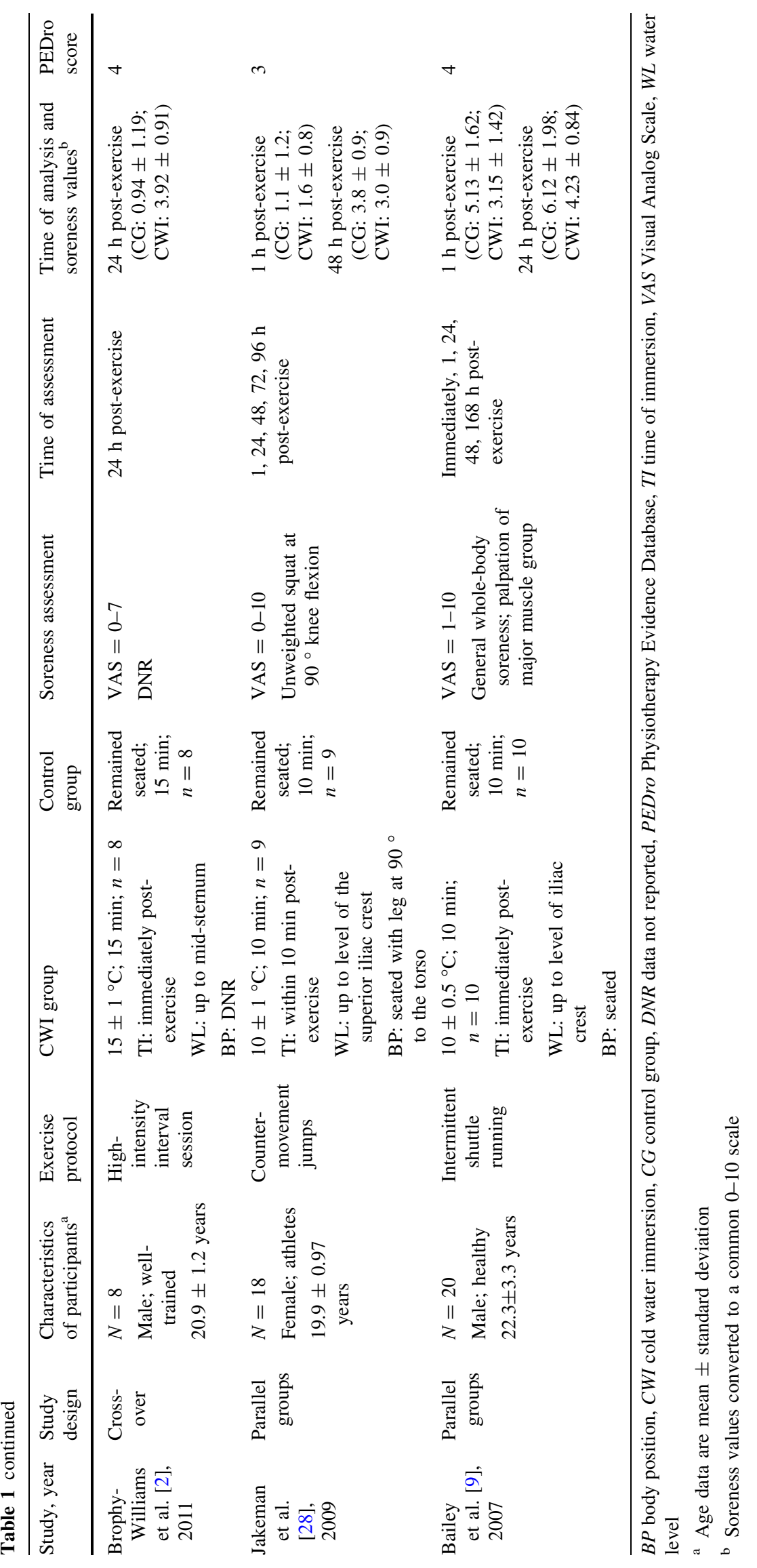


Fig. 3 Forest plot illustrating immersion versus passive recovery on muscle soreness (immediate effect, stratified by water temperature). $C I$ water immersion the effects of cold water confidence interval, $C W I$ cold

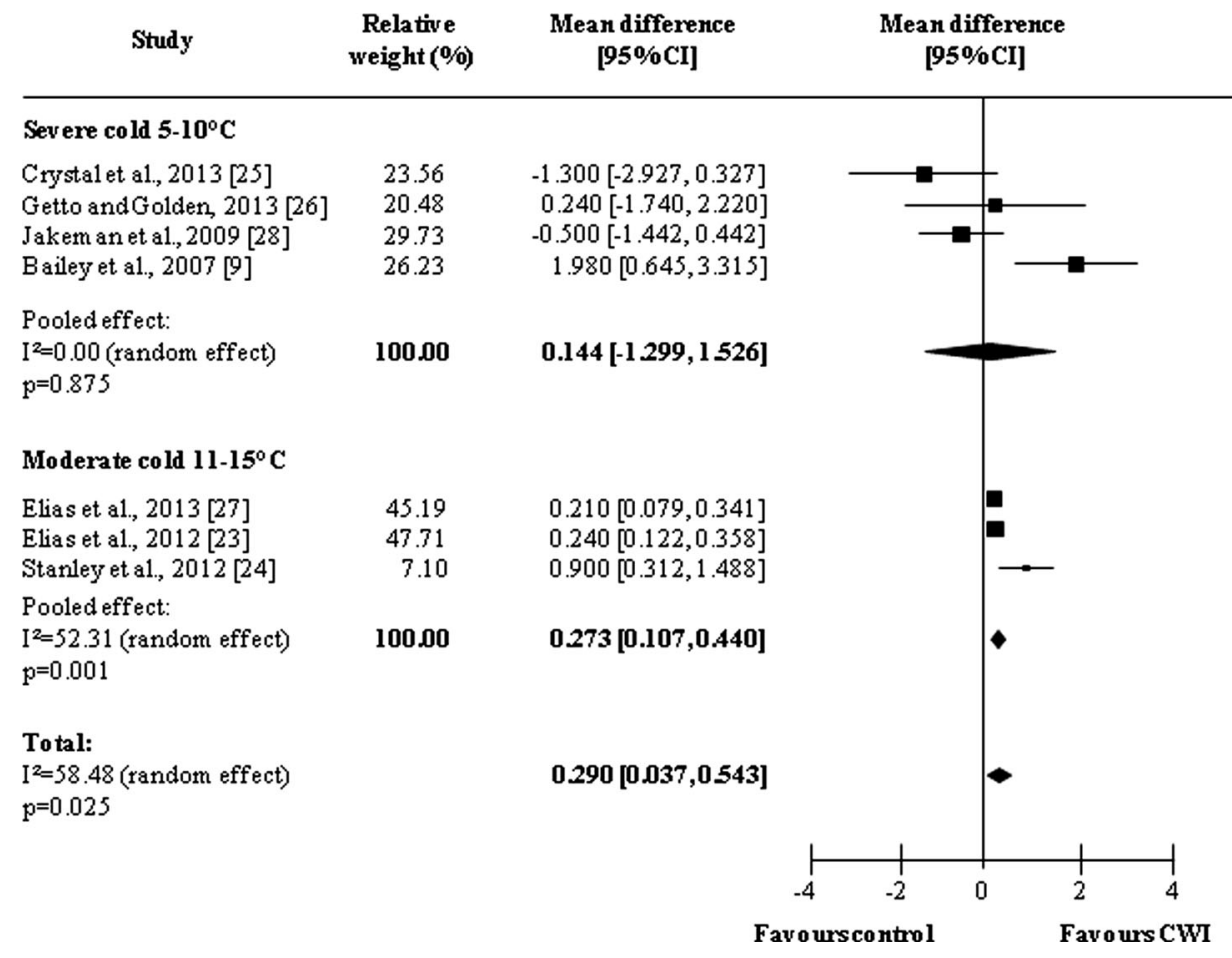

immediate effects shows a significant pooled effect for CWI (MD $=0.290,95 \%$ CI 0.037, 0.543; $p=0.025)$. When subgroups were analyzed, it was observed that studies using a water temperature of between 11 and $15{ }^{\circ} \mathrm{C}$ (moderate cold) produced better results than those using water between 5 and $10{ }^{\circ} \mathrm{C}$ (severe cold). Therefore, temperatures higher than $10^{\circ} \mathrm{C}$ present the best results for immediate effect on muscle soreness (severe cold: $\mathrm{MD}=0.144,95 \% \mathrm{CI}-1.299,1.526, p=0.875$; moderate cold: $\mathrm{MD}=0.273,95 \%$ CI 0.107, 0.440, $p=0.001$ ).

Eight studies [2, 9, 22, 23, 25-28] were included in the analysis of water temperature on delayed effects, with pooled results showing a tendency similar to immediate effect results (Fig. 4). Overall pooled results, independent of water temperature, showed a statistically significant difference in favor of CWI $(\mathrm{MD}=0.315,95 \%$ CI 0.048 , $0.581 ; p=0.021)$. Analysis of subgroups revealed that water at temperatures of between 11 and $15{ }^{\circ} \mathrm{C}$ were more effective than temperatures of $\leq 10^{\circ} \mathrm{C}$ (severe cold: $\mathrm{MD}=0.057,95 \% \mathrm{CI}-1.483,1.598, p=0.942$; moderate cold: $\mathrm{MD}=0.317,95 \%$ CI $0.102,0.532, p=0.004)$.

\subsection{Analysis of Immersion Time}

Figure 5 shows the results of analysis of immediate effect in relation to immersion time. Overall, CWI was more effective than the control condition $(\mathrm{MD}=0.290,95 \% \mathrm{CI}$ $0.037,0.543 ; p=0.025)$. Three categories were used for subgroup analysis: short, medium, and longer immersion. The medium immersion category, which had duration of between 10 and $15 \mathrm{~min}$, was responsible for the best results in terms of immediate effects. Although there is only one study featuring 'longer immersion' [25] it was observed that the effect of such treatment was less beneficial than passive recovery (short immersion: $\mathrm{MD}=0.646,95 \%$ $-0.360,1.652, \quad p=0.208$; medium immersion: $\mathrm{MD}=0.227,95 \% \quad 0.139,0.314, \quad p<0.001 ; \quad$ longer immersion: $\mathrm{MD}=-1.300,95 \%$ CI $-2.927,0.327$, $p=0.117)$.

In terms of delayed effects, the overall pooled effects of CWI described in the eight studies analyzed were positive $(\mathrm{MD}=0.315,95 \%$ CI 0048, 0581, $p=0.021$ ) (Fig. 6). As with the immediate effects, an immersion time of between 11 and 15 min produced the best results (short immersion: $\mathrm{MD}=0.728,95 \%$ CI $-0.561,2.017$, $p=0.268$; medium immersion: $\mathrm{MD}=0.317,95 \% 0.102$, $0.532, p=0.004$; longer immersion: $\mathrm{MD}=-2.200,95 \%$ CI $-4.169,-0.231, p=0.029)$.

\section{Discussion}

The results of the meta-analysis of CWI as a post-exercise recovery technique and reliever of muscle soreness were statistically consistent, and revealed the following findings: (1) independent of time and temperature, CWI produces 
Fig. 4 Forest plot illustrating the effects of cold water immersion versus passive recovery on muscle soreness (delayed effect, stratified by water temperature). $C I$ confidence interval, $C W I$ cold water immersion
Fig. 5 Forest plot illustrating the effects of cold water immersion versus passive recovery on muscle soreness (immediate effect, stratified by water immersion time). $C I$ confidence interval, $C W I$ cold water immersion

\begin{tabular}{cccc} 
Study & $\begin{array}{c}\text { Relative } \\
\text { weight (\%) }\end{array}$ & Mean difference & Mean difference \\
& {$[95 \% \mathrm{CI}]$} & {$[95 \% \mathrm{CI}]$} \\
\hline
\end{tabular}

\section{Severe cold $5-10^{\circ} \mathrm{C}$}

Crystal et al., 2013 [25]

Getto andGolden, 2013 [26]

Jakem an et al., 2009 [28]

21.40

22.20

30.01

Bailey et al., 2007 [9]

26.39

$-2.200[-4.169,-0.231]$

$-0.950[-2.814,0.914]$

$0.800[-0.032,1.632]$

$1.890[0.557,3.223]$

Pooled effect:

$I^{2}=21.71$ (random effect) $\quad 100.00$ $\mathrm{p}=0.942$

$0.057[-1.483,1598]$

Moderate cold $11-15^{\circ} \mathrm{C}$

Elias et al., 2013 [27]

Elias et al., 2012 [23]

Brophy-William s et al., 2011 [2]

Leeder et a1., 2015 [22]

48.83

Pooled effect:

$I^{2}=53.20$ (random effect) $\quad 100.00$

$\mathrm{p}=0.004$

Total:

$I^{2}=63.64$ (random effect)

$\mathrm{p}=0.021$

$0.280[0.172,0.388]$

$0.450[0.370,0.530]$

$-0.980[-2.018,0.058]$

$0.650[-1.460,2.760]$

0.317 [0.102,0532]

0.315 [0.048,0581]

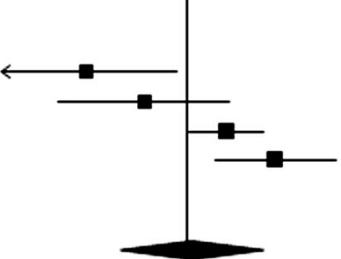


Fig. 6 Forest plot illustrating the effects of cold water immersion versus passive recovery on muscle soreness (delayed effect, stratified by water immersion time). $C I$ confidence interval, $C W I$ cold water immersion

\begin{tabular}{|c|c|}
\hline Study & $\begin{array}{l}\text { Relative } \\
\text { weight (o) }\end{array}$ \\
\hline \multicolumn{2}{|l|}{ Short immersio $\mathrm{n} \leq 10$ minutes } \\
\hline $\begin{array}{l}\text { Getto andGolden, } 2013 \text { [26] } \\
\text { Jakem an et a1., } 2009 \text { [28] } \\
\text { Bailey et a1., 2007 [9] }\end{array}$ & $\begin{array}{l}24.73 \\
42.20 \\
33.08\end{array}$ \\
\hline $\begin{array}{l}\text { Pooled effect: } \\
I^{2}=24.45 \text { (random effect) } \\
\mathrm{p}=0.268\end{array}$ & 100.00 \\
\hline \multicolumn{2}{|c|}{ Medium immersion $11-15$ minutes } \\
\hline $\begin{array}{l}\text { Elias et al., } 2013 \text { [27] } \\
\text { Elias et al., } 2012 \text { [23] }\end{array}$ & $\begin{array}{l}46.20 \\
48.83\end{array}$ \\
\hline $\begin{array}{l}\text { Brophy-William s et a1, } 2011 \\
\text { Leeder et a1., } 2015 \text { [22] }\end{array}$ & 2] $\begin{array}{l}3.95 \\
1.01\end{array}$ \\
\hline $\begin{array}{l}\text { Pooled effect: } \\
I^{2}=67.77 \text { (random effect) } \\
p=0.004\end{array}$ & 10000 \\
\hline Lo nger immersion $16-20$ minu & utes \\
\hline Crystal et a1., 2013 [25] & \\
\hline $\begin{array}{l}\text { Pooled effect: } \\
I^{2}=\text { Not applicable } \\
\mathrm{p}=0.029\end{array}$ & 100.00 \\
\hline $\begin{array}{l}\text { Total: } \\
I^{2}=63.64 \text { (random effect) } \\
\mathrm{p}=0.021\end{array}$ & \\
\hline
\end{tabular}

10000 generally positive results in terms of both immediate and delayed effects; (2) immersion in water at temperatures between 11 and $15{ }^{\circ} \mathrm{C}$ appeared to produce a greater reduction of muscle soreness after exercise; and (3) 11-15 min appeared to be the optimal immersion time for the relief of muscle soreness caused by exercise.

The findings regarding CWI, independent of immersion temperature and time, are in accordance with previous reviews, such as the studies by Leeder et al. [8] and Bleakley et al. [12]. The authors claim that the technique is capable of altering blood flow, thereby causing vasoconstriction and redirection of the blood [14]. The real effects of CWI have not yet been fully elucidated [4], but it has been speculated that this technique is able to reduce lymphatic and capillary cell permeability [14, 29], resulting in decreased fluid diffusion, which may assist in the reduction of the inflammatory process caused by exercise [14].

This technique can also reduce nerve velocity conduction and muscle spasm [18]. This mechanism is derived from decreasing the transmission rate of neurons due to reduction in acetylcholine production [14]. Studies also suggest that CWI can affect the exchange between $\mathrm{Ca}^{2+}$ and $\mathrm{Na}^{2+}$ in neural cells [18], which may lead to a delay in action potential generation [30], contraction speed, and force-generating capacity [31], reducing the dynamic contractile force by $4-6 \%$ for every $1{ }^{\circ} \mathrm{C}$ reduction in muscle temperature [6]. These changes may result in decreased sports performance if the exercise is performed immediately after CWI [14, 15, 32].

The reduction in soreness could also be explained by the same factors that are associated with analgesia, which occurs in response to the reduction in the pain-spasm cycle [32]. Algafly and George [18] showed that cooling that is able to reduce the skin temperature by $10-13{ }^{\circ} \mathrm{C}$ can promote a reduction of 10-33\% in NVC. However, the same temperature reduction is considered beneficial for analgesia.

While the effects of CWI have been widely investigated, opinions vary with regard to method of application [16]. The variance in effects caused by temperature change observed in this review revealed that CWI was more effective in terms of both immediate and delayed effects when temperatures were in the 'moderate cold' category $\left(11-15{ }^{\circ} \mathrm{C}\right)$. The benefits of 'moderate cold' temperatures have not been discussed in clinical studies. However, it has been shown that immersion at very low temperatures can 
cause adverse effects that are interpreted by the body as noxious stimuli [33], such as nerve damage [34]. Regarding water temperature, a beneficial and safe lower limit for skin temperature remains uncertain [34]. It has been suggested that severe cold can produce hyperventilation, leading to blood acidosis [14]. In extreme situations, severe cold can cause loss of consciousness or even death [14].

A study by Getto and Golden [26] claims that short immersions are less efficient at lessening muscle soreness caused by exercise, due to limited muscle temperature reduction [5]. Such a statement confirms the findings of this study, which indicate that medium immersions of between 11 and 15 min produce better results than short immersions ( $\leq 10 \mathrm{~min}$ ). Additionally, during the immediate effect, there is the presence of the category 'longer immersion', which is responsible for the worst results. Although pooled results were not available for this category due to an insufficient number of studies $(n=1)$, it compared unfavorably with passive recovery. The study in question [25] considered the use of a very low temperature $\left(5^{\circ} \mathrm{C}\right)$ for $20 \mathrm{~min}$. Davis and Pope [35] claimed that an application of $10 \mathrm{~s}$ at low temperatures was required for CWI to produce harmful stimulation and pain. Such effects can be exacerbated during long immersion conditions [36, 37], causing a greater depression of sensory and motor NCV, and producing superficial nerve damage [31]. Vaile et al. [38] state that the best responses result from faster cooling, but there are difficulties in defining the limit and exact magnitude.

Accordingly, it is important to analyze studies by subdivisions of water temperature and immersion time. The limited number of studies, however, does not allow the implementation of closed protocol comparisons. The relationship between the two variables could allow objective inferences about the most effective recovery model to be used.

As in the study by Crystal et al. [25], which showed that exposure to a low temperature for longer durations compared unfavorably with passive recovery, other studies have reported unfavorable results for CWI for various other reasons. Getto and Golden [26] used a scale that involved six different points, including the low back, to analyze soreness. This type of evaluation considers a larger number of soreness points than other studies and can result in participant confusion in relation to the effects of CWI. Jakeman et al. [28] found no significant difference between CWI and a control group following counter-movement jumps. These authors justify the absence of results favorable to CWI based on variation of time related to analgesic mechanisms, which can last from a few minutes to hours, and further report these mechanisms as uncertain [28]. The main methodological difference that can be considered as a hypothesis for divergence in the results refers to the sample. Evidence suggests that there are sex-specific physiological responses after body cooling [39]. In our systematic review, studies with female participants were not found to have differences favoring CWI [26, 28].

Overall, the studies selected for this review show similar models of inducing stress, represented by physical activities featuring high intensity of effort. This factor is relevant to data interpretation, as different types of stress provide different outcomes, as previously explained. For example, the characteristics of injuries induced in localized eccentric exercise can differ from those sustained during sporting activities, and respond differently to the application of CWI [7, 40].

To the authors' knowledge, this is the first systematic review and meta-analysis to investigate the effects of different CWI procedures, namely variations in water temperature and immersion time. The strengths of this systematic review relate mainly to the rationale of the study, which aims to analyze the dose-response relationship, which is still poorly investigated in studies of this nature. Despite the statistically significant effects on muscle soreness favoring CWI, the small effect size of the intervention should be considered. It is possible to observe a decrease in muscle soreness of approximately $5 \%$ in the best situation (upper limit of delayed effect for moderate cold and medium immersion). Although this reduction may be small, there is no evidence to indicate what the minimal clinical difference detected is in this population, especially in athletes.

One of the limitations of the study is the poor methodological quality of the studies included. Future trials should be attentive to the criteria for the development of a highquality study, which would result in surveys with greater scientific evidence. Other limitations are related to the sample heterogeneity, due to no restrictions in the search strategy and the investigation of a single outcome. Although this is a key outcome in the recovery of an athlete, further studies should consider the dose-response effect of CWI on other markers of muscle damage, such as performance, in order to identify the best CWI recovery strategy based on different and relevant factors.

\section{Conclusion}

The findings of the present study suggest that CWI may be slightly better than passive recovery in the management of muscle soreness. The results also demonstrate the presence of a dose-response relationship, indicating that CWI provides the best results at temperatures between 11 and $15^{\circ} \mathrm{C}$ for 11-15 min. The low quality of the included studies and the small size of the intervention effect should be considered. Higher-quality studies are needed to investigate whether the dose-response relationship of the results can be reliably reproduced. 
The findings of the study allow athletes using CWI to have a better understanding of the technique. For those applying CWI, it enables the use of improved logistics and therefore results in lower costs due to the most effective use of immersion time and water temperature.

\section{Compliance with Ethical Standards}

Funding This work was supported by The São Paulo Research Foundation-FAPESP master degree scholarship (Grant Number 2013/12474-7) and FAPESP research internships abroad (Grant Number 2014/0338-5), and by the National Council for Scientific and Technological Development-CNPq (Grant Number 482749/2012-1).

Conflicts of interest Aryane Flauzino Machado, Paulo Henrique Ferreira, Jéssica Kirsch Micheletti, Aline Castilho de Almeida, Ítalo Ribeiro Lemes, Franciele Marques Vanderlei, Jayme Netto Junior, and Carlos Marcelo Pastre declare that they have no conflicts of interest relevant to the content of this review.

\section{References}

1. Bastos FN, Vanderlei LCM, Nakamura FY, et al. Effects of cold water immersion and active recovery on post-exercise heart rate variability. Int J Sports Med. 2012;33(11):873-9.

2. Brophy-Williams N, Landers G, Wallman K. Effect of immediate and delayed cold water immersion after a high intensity exercise session on subsequent run performance. J Sports Sci Med. 2011:665-670.

3. Pournot H, Bieuzen F, Duffield R, et al. Short term effects of various water immersions on recovery from exhaustive intermittent exercise. Eur J Appl Physiol. 2011;111(7):1287-95.

4. Broatch JR, Petersen A, Bishop DJ. Postexercise cold water immersion benefits are not greater than the placebo effect. Med Sci Sports Exerc. 2014;46(11):2139-47.

5. Bleakley CM, Davison GW. What is the biochemical and physiological rationale for using cold-water immersion in sports recovery? A systematic review. $\mathrm{Br} \mathrm{J}$ Sports Med. 2010;44(3):179-87.

6. Costello JT, Culligan K, Selfe J, Donnelly AE. Muscle, skin and core temperature after $-110{ }^{\circ} \mathrm{C}$ cold air and $8{ }^{\circ} \mathrm{C}$ water treatment. PLoS One. 2012;7(11):e48190.

7. Bieuzen F, Bleakley CM, Costello JT. Contrast water therapy and exercise induced muscle damage: a systematic review and metaanalysis. PLoS One. 2013;8(4):e62356.

8. Leeder J, Gissane C, van Someren K, et al. Cold water immersion and recovery from strenuous exercise: a meta-analysis. $\mathrm{Br} \mathrm{J}$ Sports Med. 2012;46(4):233-40.

9. Bailey DM, Erith SJ, Griffin PJ, et al. Influence of cold-water immersion on indices of muscle damage following prolonged intermittent shuttle running. J Sports Sci. 2007;25(11):1163-70.

10. Glasgow PD, Ferris R, Bleakley CM. Cold water immersion in the management of delayed-onset muscle soreness: is dose important? A randomised controlled trial. Phys Ther Sport. 2014;15(4):228-33.

11. Leal Junior EC, de Godoi V, Mancalossi JL, et al. Comparison between cold water immersion therapy (CWIT) and light emitting diode therapy (LEDT) in short-term skeletal muscle recovery after high-intensity exercise in athletes-preliminary results. Lasers Med Sci. 2011;26(4):493-501.

12. Bleakley C, Mcdonough S, Gardner E, et al. Cold-water immersion (cryotherapy) for preventing and treating muscle soreness after exercise. Cochrane Database Syst Rev. 2012;2:CD008262.

13. White GE, Wells GD. Cold-water immersion and other forms of cryotherapy: physiological changes potentially affecting recovery from high-intensity exercise. Extrem Physiol Med. 2013;2(1):26.

14. Wilcock IM, Cronin JB, Hing WA. Physiological response to water immersion: a method for sport recovery. Sports Med. 2006;36(9):1-18.

15. García-Manso JM, Rodríguez-Matoso D, Rodríguez-Ruiz D, et al. Effect of cold-water immersion on skeletal muscle contractile properties in soccer players. Am J Phys Med Rehabil. 2011;90(5):356-63.

16. Pastre CM, Bastos FN, Netto J Jr, et al. Métodos de recuperação pós-exercício: uma revisão sistemática. Rev Bras Med Esporte. 2009; 15:138-44.

17. Chesterton LS, Foster NE, Ross L. Skin temperature response to cryotherapy. Arch Phys Med Rehabil. 2002;83(4):543-9.

18. Algafly AA, George KP. The effect of cryotherapy on nerve conduction velocity, pain threshold and pain tolerance. $\mathrm{Br} \mathrm{J}$ Sports Med. 2007;41(6):365-9.

19. Maher CG, Sherrington C, Robert D, et al. Research report reliability of the PEDro scale for rating quality of randomized. Phys Ther. 2003;83:713-21.

20. Macedo LG, Elkins MR, Maher CG, et al. There was evidence of convergent and construct validity of Physiotherapy Evidence Database quality scale for physiotherapy trials. J Clin Epidemiol. 2010;63(8):920-5.

21. Bleakley CM, Glasgow PD, Philips P, et al. Management of acute soft tissue injury using protection, rest, ice, compression, and elevation: recommendations from the Association of Chartered Physiotherapists in Sports and Exercise Medicine (ACPSM). London: Association of Chartered Physiotherapists in Sports and Exercise Medicine (ACPS); 2010.

22. Leeder JDC, van Someren KA, Bell PG, et al. Effects of seated and standing cold water immersion on recovery from repeated sprinting. J Sports Sci. 2015;33(15):1544-52.

23. Elias GP, Varley MC, Wyckelsma VL, et al. Effects of water immersion on posttraining recovery in Australian footballers. Int J Sports Physiol Perform. 2012;7(4):357-66.

24. Stanley J, Buchheit M, Peake JM. The effect of post-exercise hydrotherapy on subsequent exercise performance and heart rate variability. Eur J Appl Physiol. 2012;112(3):951-61.

25. Crystal NJ, Townson DH, Cook SB, et al. Effect of cryotherapy on muscle recovery and inflammation following a bout of damaging exercise. Eur J Appl Physiol. 2013;113(10):2577-86.

26. Getto CN, Golden G. Comparison of active recovery in water and cold-water immersion after exhaustive exercise. Athl Train Sport Heal Care. 2013;5(4):169-76.

27. Elias GP, Wyckelsma VL, Varley MC, et al. Effectiveness of water immersion on postmatch recovery in elite professional footballers. Int J Sports Physiol Perform. 2013;8(3):243-54.

28. Jakeman JR, Macrae R, Eston R. A single 10-min bout of coldwater immersion therapy after strenuous plyometric exercise has no beneficial effect on recovery from the symptoms of exerciseinduced muscle damage. Ergonomics. 2009;52(4):456-60.

29. Ascensão A, Leite M, Rebelo AN, et al. Effects of cold water immersion on the recovery of physical performance and muscle damage following a one-off soccer match. J Sports Sci. 2011;29(3):217-25.

30. Reid G, Babes A, Pluteanu F. A cold and menthol-activated current in rate dorsal root ganglion neurones: properties and role in cold transduction. J Physiol. 2002;545:595-614.

31. Herrera E, Sandoval MC, Camargo DM, et al. Motor and sensory nerve conduction are affected differently by ice pack, ice masage, and cold water immersion. Phys Ther. 2010;90(4):581-91. 
32. Halson SL, Quod MJ, Martin DT, et al. Physiological responses to cold water immersion following cycling in the heat. Int J Sport Physiol Perform. 2008;3:331-46.

33. Sellwood KL, Brukner P, Williams D, et al. Ice-water immersion and delayed-onset muscle soreness: a randomised controlled trial. Br J Sports Med. 2007;41(6):392-7.

34. Costello JT, McInerney CD, Bleakley CM, et al. The use of thermal imaging in assessing skin temperature following cryotherapy: a review. J Therm Biol. 2012;37:103-10.

35. Davis KD, Pope GE. Noxious cold evokes multiple sensations with distinct time courses. Pain. 2002;98(1-2):179-85.

36. Yeargin SW, Casa DJ, McClung JM, et al. Body cooling between two bouts of exercise in the heat enhances subsequent performance. J Strength Cond Res. 2006;20(2):383-9.
37. Versey NG, Halson SL, Dawson BT. Water immersion recovery for athletes: effect on exercise performance and practical recommendations. Sports Med. 2013;43(11):1101-30.

38. Vaile J, Halson S, Gill N, Dawson B. Effect of hydrotherapy on recovery from fatigue. Int J Sports Med. 2008;29(7):539-44.

39. Solianik R, Skurvydas A, Pukenas K, et al. Comparison of the effects of whole-body cooling during fatiguing exercise in males and females. Cryobiology. 2015;71(1):112-8.

40. Pointon M, Duffield R, Cannon J, et al. Cold application for neuromuscular recovery following intense lower-body exercise. Eur J Appl Physiol. 2011;111(12):2977-86. 